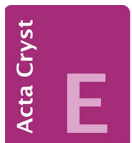

CRYSTALLOGRAPHIC COMMUNICATIONS

ISSN 2056-9890

\section{Crystal structure of azimsulfuron}

\section{Youngeun Jeon, Jineun Kim, ${ }^{*}$ Eunjin Kwon and Tae Ho}

Kim*

Department of Chemistry and Research Institute of Natural Sciences, Gyeongsang National University, Jinju 660-701, Republic of Korea. *Correspondence e-mail:

thkim@gnu.ac.kr, jekim@gnu.ac.kr

Received 3 June 2015; accepted 6 June 2015

Edited by P. C. Healy, Griffith University, Australia

The title compound \{systematic name: 1-(4,6-dimethoxypyrimidin-2-yl)-3-[1-methyl-4-(2-methyl-2 $H$-tetrazol-5-yl)pyrazol-5-ylsulfonyl]urea\}, $\mathrm{C}_{13} \mathrm{H}_{16} \mathrm{~N}_{10} \mathrm{O}_{5} \mathrm{~S}$, is a sulfonylurea herbicide. In this compound, the dihedral angles between the planes of the central pyrazole and the terminal dimethoxypyrimidine and tetrazole rings are $79.10(8)$ and $17.21(16)^{\circ}$, respectively. In the crystal, $\mathrm{N}-\mathrm{H} \cdots \mathrm{O}$ hydrogen bonds link adjacent molecules, forming $R_{2}^{2}(8)$ inversion dimers. In addition, weak $\mathrm{C}-\mathrm{H} \cdots \mathrm{O}$ and $\mathrm{C}-\mathrm{H} \cdots \mathrm{N}$ hydrogen bonds and weak $\pi-\pi$ interactions [ring centroid separation $=$ $3.8255(12) \AA]$ are present, resulting in a three-dimensional architecture.

Keywords: crystal structure; azimsulfuron; hydrogen bonding; herbicide.

CCDC reference: 1405357

\section{Related literature}

For information on the herbicidal properties of the title compound, see: Valle et al. (2006); Boschin et al. (2007). For a related crystal structure, see: Chopra et al. (2004).<smiles>COc1cc(OC)nc(NC(=O)NS(=O)(=O)c2c(-c3nnn(C)n3)cnn2C)n1</smiles>

\section{Experimental}

2.1. Crystal data

$\mathrm{C}_{13} \mathrm{H}_{16} \mathrm{~N}_{10} \mathrm{O}_{5} \mathrm{~S}$

$M_{r}=424.42$

Triclinic, $P \overline{1}$

$a=8.5884$ (7) ̊

$b=9.9165(7) \AA$

$c=12.2788(13) \AA$

$\alpha=73.190(5)^{\circ}$

$\beta=75.819$ (4)

\subsection{Data collection}

Bruker APEXII CCD

diffractometer

Absorption correction: multi-scan

(SADABS; Bruker, 2013)

$T_{\min }=0.909, T_{\max }=0.979$

$\gamma=66.374(3)^{\circ}$

$V=907.16(14) \AA^{3}$

$Z=2$

Mo $K \alpha$ radiation

$\mu=0.23 \mathrm{~mm}^{-1}$

$T=173 \mathrm{~K}$

$0.42 \times 0.10 \times 0.09 \mathrm{~mm}$

\subsection{Refinement}

$R\left[F^{2}>2 \sigma\left(F^{2}\right)\right]=0.045$

$w R\left(F^{2}\right)=0.118$

$S=1.04$

4123 reflections

266 parameters

$\mathrm{H}$-atom parameters constrained

$\Delta \rho_{\max }=0.56{\mathrm{e} \AA^{-3}}^{-3}$

$\Delta \rho_{\min }=-0.49{\mathrm{e} \AA^{-3}}^{-3}$

Table 1

Hydrogen-bond geometry $\left(\AA{ }^{\circ}\right)$.

\begin{tabular}{|c|c|c|c|c|}
\hline$D-\mathrm{H} \cdots A$ & $D-\mathrm{H}$ & $\mathrm{H} \cdots A$ & $D \cdots A$ & $D-\mathrm{H} \cdots A$ \\
\hline $\mathrm{N} 3-\mathrm{H} 3 N \cdots \mathrm{O} 3^{\mathrm{i}}$ & 0.88 & 2.09 & $2.877(2)$ & 149 \\
\hline $\mathrm{C} 1-\mathrm{H} 1 A \cdots \mathrm{O} 3^{\mathrm{ii}}$ & 0.98 & 2.55 & $3.381(3)$ & 143 \\
\hline $\mathrm{C} 1-\mathrm{H} 1 C \cdots \mathrm{O} 4^{\mathrm{iii}}$ & 0.98 & 2.44 & $3.225(3)$ & 137 \\
\hline $\mathrm{C} 11-\mathrm{H} 11 A \cdots \mathrm{N} 9^{\mathrm{iv}}$ & 0.98 & 2.57 & $3.357(3)$ & 137 \\
\hline $\mathrm{C} 13-\mathrm{H} 13 B \cdots \mathrm{N} 6^{\mathrm{v}}$ & 0.98 & 2.62 & $3.533(3)$ & 155 \\
\hline
\end{tabular}

Data collection: APEX2 (Bruker, 2013); cell refinement: SAINT (Bruker, 2013); data reduction: $S A I N T$; program(s) used to solve structure: SHELXS97 (Sheldrick, 2008); program(s) used to refine structure: SHELXL2013 (Sheldrick, 2015); molecular graphics: SHELXTL (Sheldrick, 2008); software used to prepare material for publication: SHELXTL.

\section{Acknowledgements}

This research was supported by the Basic Science Research Program through the National Research Foundation of Korea (NRF) funded by the Ministry of Education, Science and Technology (No. 2014R1A1A4A01009105).

Supporting information for this paper is available from the IUCr electronic archives (Reference: HG5444). 


\section{data reports}

\section{References}

Boschin, G., D’Agostina, A., Antonioni, C., Locati, D. \& Arnoldi, A. (2007). Chemosphere, 68, 1312-1317.

Bruker (2013). APEX2, SAINT and SADABS. Bruker AXS Inc., Madison, Wisconsin, USA.
Chopra, D., Mohan, T. P., Rao, K. S. \& Guru Row, T. N. (2004). Acta Cryst. E60, o2418-o2420.

Sheldrick, G. M. (2008). Acta Cryst. A64, 112-122.

Sheldrick, G. M. (2015). Acta Cryst. C71, 3-8.

Valle, A., Boschin, G., Negri, M., Abbruscato, P., Sorlini, C., D'Agostina, A. \& Zanardini, E. (2006). J. Appl. Microbiol. 101, 443-452. 


\section{supporting information}

Acta Cryst. (2015). E71, o470-o471 [doi:10.1107/S2056989015010968]

\section{Crystal structure of azimsulfuron}

\section{Youngeun Jeon, Jineun Kim, Eunjin Kwon and Tae Ho Kim}

\section{S1. Comment}

Azimsulfuron [systematic name: 1-(4,6-dimethoxypyrimidin-2-yl)-3-[1-methyl-4-(2-methyl-2H-tetrazol-5-yl)pyrazol-5ylsulfonyl]urea] is a sulfonylurea herbicide, a group of pesticides widely used all over the world for controlling weeds in several crops, rice, wheat, maize, barley, sugar beet, and tomato (Valle et al., 2006). However, until now its crystal structure has not been reported. In the title compound (Fig. 1), the dihedral angles between the planes of the central pyrazol and the terminal dimethoxypyrimidinyl and tetrazol rings are $79.10(8)$ and $17.21(16)^{\circ}$, respectively. All bond lengths and bond angles are normal and comparable to those observed in similar crystal structures (Chopra et al., 2004). In the crystal structure (Fig. 2), molecules are linked by a pairs of $\mathrm{N}-\mathrm{H} \cdots \mathrm{O}$ hydrogen bonds(Table 1), forming inversion dimmers with an $R_{2}{ }^{2}(8)$ ring motif. In addition, weak $\mathrm{C}-\mathrm{H} \cdots \mathrm{O}$ and $\mathrm{C}-\mathrm{H} \cdots \mathrm{N}$ hydrogen bonding and weak intermolecular $\pi-\pi$ interactions between the terminal tetrazol ring systems $\left[\mathrm{Cg} 2 \cdots \mathrm{Cg} 2^{\mathrm{i}}=3.8255\right.$ (12) $\left.\AA\right]$ are present $(\mathrm{Cg} 2$ is the centroid of the N7-N8-N9-N10-C12 ring) [for symmetry codes: (i), $-x,-y+1,-z+1$ ].

\section{S2. Experimental}

The title compound was purchased from the Dr. Ehrenstorfer $\mathrm{GmbH}$ Company. Slow evaporation of a solution in $\mathrm{CH}_{2} \mathrm{Cl}_{2}$ gave single crystals suitable for X-ray analysis.

\section{S3. Refinement}

All H-atoms were positioned geometrically and refined using a riding model with $\mathrm{d}(\mathrm{N}-\mathrm{H})=0.88 \AA, U_{\text {iso }}=1.2 U_{\text {eq }}(\mathrm{C})$ for urea $\mathrm{N}-\mathrm{H}, \mathrm{d}(\mathrm{C}-\mathrm{H})=0.98 \AA, U_{\text {iso }}=1.5 U_{\text {eq }}(\mathrm{C})$ for methyl group, $\mathrm{d}(\mathrm{C}-\mathrm{H})=0.95 \AA, U_{\text {iso }}=1.2 U_{\text {eq }}(\mathrm{C})$ for aromatic $\mathrm{C}-$ $\mathrm{H}$. 


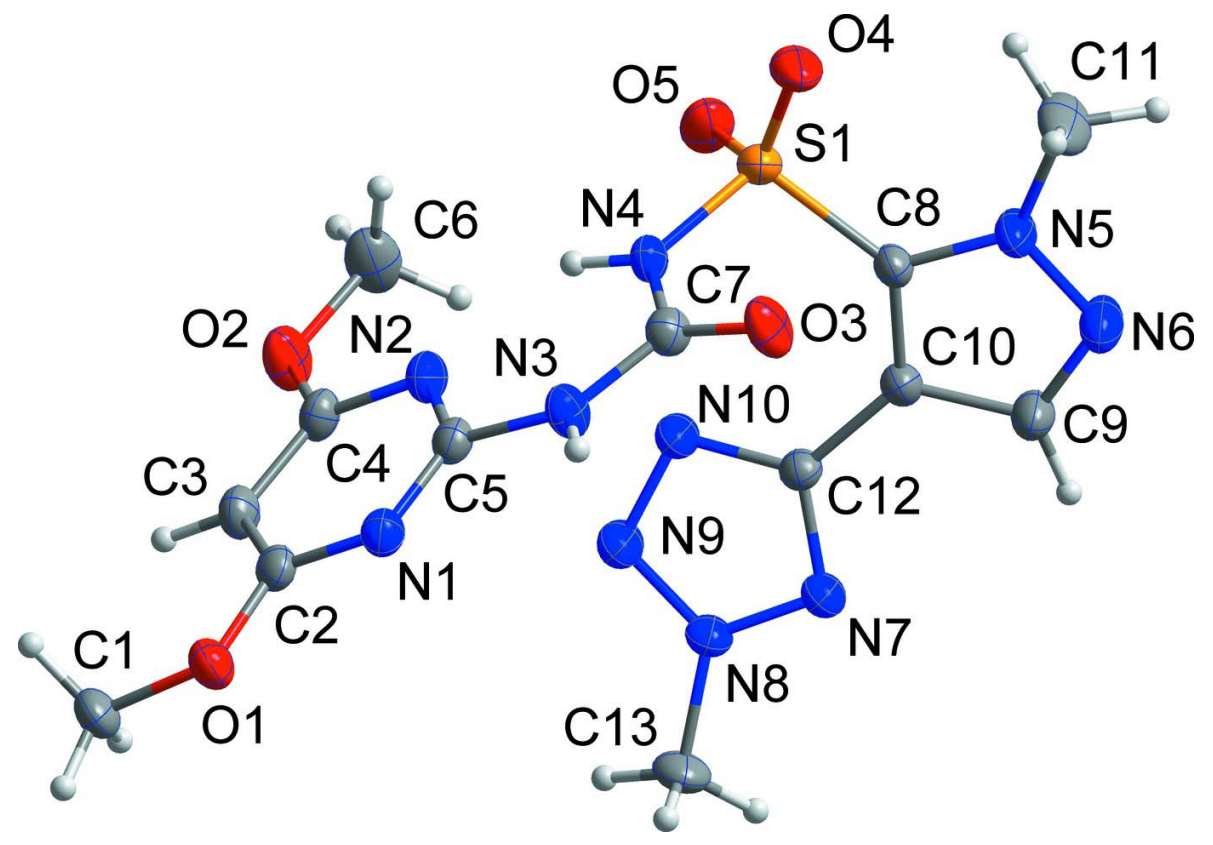

\section{Figure 1}

The asymmetric unit of the title compound with the atom numbering scheme. Displacement ellipsoids are drawn at the $50 \%$ probability level. $\mathrm{H}$ atoms are shown as small spheres of arbitrary radius. 


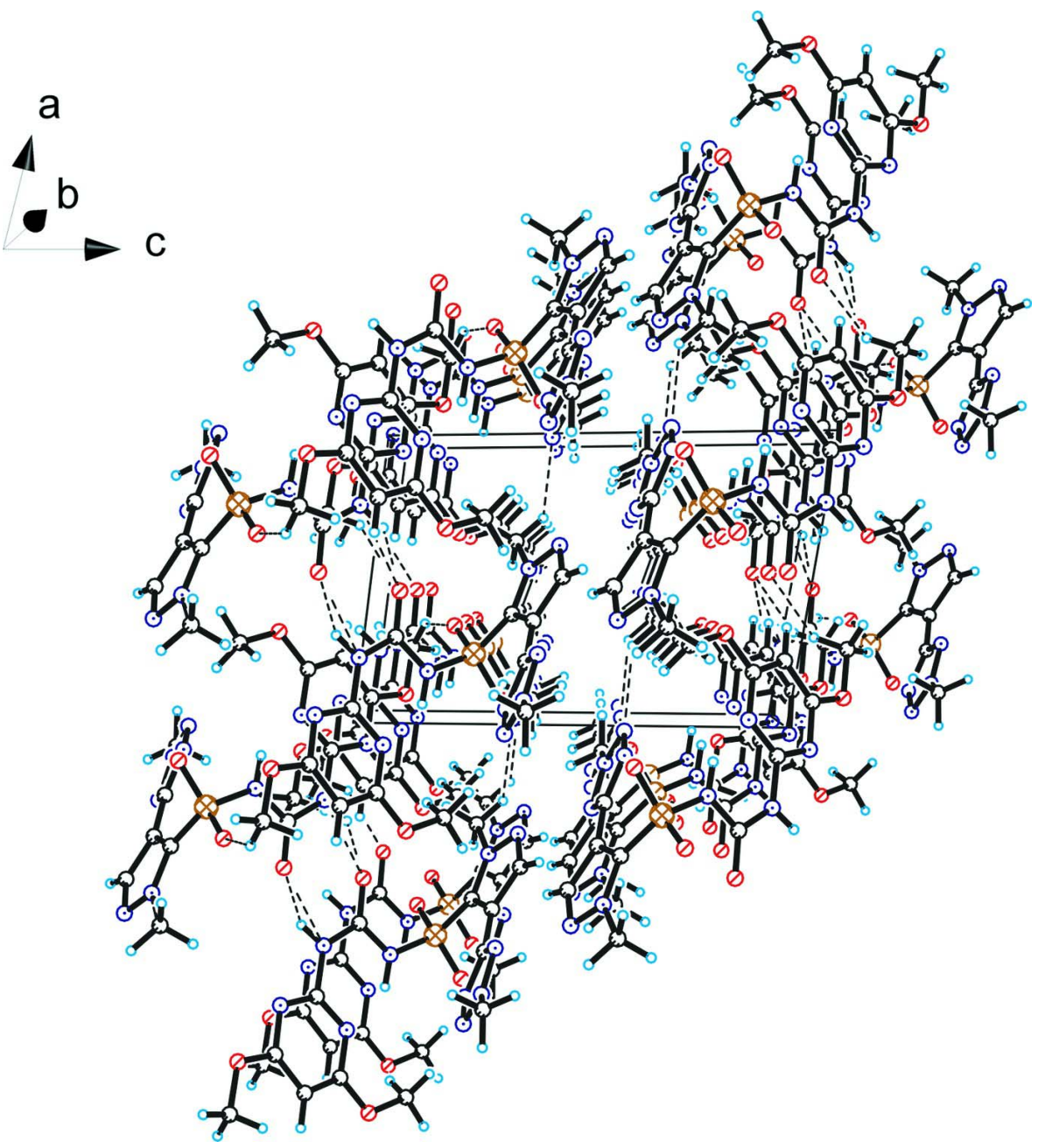

Figure 2

Crystal packing viewed along the $b$ axis. The hydrogen bonds are shown as dashed lines.

\section{1-(4,6-Dimethoxypyrimidin-2-yl)-3-[1-methyl-4-(2-methyl-2H-tetrazol-5-yl)pyrazol-5-ylsulfonyl]urea}

\section{Crystal data}

$\mathrm{C}_{13} \mathrm{H}_{16} \mathrm{~N}_{10} \mathrm{O}_{5} \mathrm{~S}$

$M_{r}=424.42$

Triclinic, $P \overline{1}$

$a=8.5884(7) \AA$

$b=9.9165(7) \AA$

$c=12.2788(13) \AA$

$\alpha=73.190(5)^{\circ}$

$\beta=75.819(4)^{\circ}$

$\gamma=66.374(3)^{\circ}$

$V=907.16(14) \AA^{3}$

\section{Data collection}

Bruker APEXII CCD

diffractometer

$\varphi$ and $\omega$ scans
$Z=2$

$F(000)=440$

$D_{\mathrm{x}}=1.554 \mathrm{Mg} \mathrm{m}^{-3}$

Mo $K \alpha$ radiation, $\lambda=0.71073 \AA$

Cell parameters from 4687 reflections

$\theta=2.3-26.9^{\circ}$

$\mu=0.23 \mathrm{~mm}^{-1}$

$T=173 \mathrm{~K}$

Block, colourless

$0.42 \times 0.10 \times 0.09 \mathrm{~mm}$

Absorption correction: multi-scan

(SADABS; Bruker, 2013)

$T_{\min }=0.909, T_{\max }=0.979$ 
16122 measured reflections

4123 independent reflections

3357 reflections with $I>2 \sigma(I)$

$R_{\text {int }}=0.036$

\section{Refinement}

Refinement on $F^{2}$

Least-squares matrix: full

$R\left[F^{2}>2 \sigma\left(F^{2}\right)\right]=0.045$

$w R\left(F^{2}\right)=0.118$

$S=1.04$

4123 reflections

266 parameters

0 restraints

$$
\begin{aligned}
& \theta_{\max }=27.5^{\circ}, \theta_{\min }=1.8^{\circ} \\
& h=-11 \rightarrow 11 \\
& k=-12 \rightarrow 12 \\
& l=-15 \rightarrow 15
\end{aligned}
$$

Hydrogen site location: inferred from neighbouring sites

$\mathrm{H}$-atom parameters constrained

$w=1 /\left[\sigma^{2}\left(F_{\mathrm{o}}^{2}\right)+(0.0543 P)^{2}+0.5222 P\right]$

where $P=\left(F_{\mathrm{o}}^{2}+2 F_{\mathrm{c}}^{2}\right) / 3$

$(\Delta / \sigma)_{\max }<0.001$

$\Delta \rho_{\max }=0.56 \mathrm{e} \AA^{-3}$

$\Delta \rho_{\min }=-0.49$ e $\AA^{-3}$

Special details

Geometry. All e.s.d.'s (except the e.s.d. in the dihedral angle between two 1.s. planes) are estimated using the full covariance matrix. The cell e.s.d.'s are taken into account individually in the estimation of e.s.d.'s in distances, angles and

\begin{tabular}{|c|c|c|c|c|}
\hline & $x$ & $y$ & $z$ & $U_{\text {iso }} * / U_{\text {eq }}$ \\
\hline $\mathrm{S} 1$ & $0.74797(6)$ & $0.79478(5)$ & 0.73248 & $0.02370(14)$ \\
\hline O1 & $1.10371(18)$ & $-0.02315(15)$ & $1.11503(12)$ & 0.0280 \\
\hline $\mathrm{O} 2$ & $1.32890(18)$ & $0.30498(17)$ & $0.81805(13)$ & $0.0356(4)$ \\
\hline $\mathrm{O} 3$ & $0.53148(17)$ & $0.65412(16)$ & $0.91451(13)$ & $0.0326(4)$ \\
\hline O4 & $0.6527(2)$ & $0.91670(16)$ & $0.78851(14)$ & $0.0379(4)$ \\
\hline O5 & 0.90400 (19) & $0.79382(17)$ & $0.65785(14)$ & $0.0361(4)$ \\
\hline N1 & $0.9372(2)$ & $0.21202(18)$ & $1.04786(14)$ & $0.0236(4)$ \\
\hline N2 & $1.0436(2)$ & $0.38255(18)$ & $0.89699(14)$ & $0.0238(4)$ \\
\hline N3 & $0.7525(2)$ & $0.44579(18)$ & $0.97760(14)$ & $0.0254(4)$ \\
\hline $\mathrm{H} 3 \mathrm{~N}$ & 0.6779 & 0.4182 & 1.0336 & $0.031^{*}$ \\
\hline N4 & $0.8032(2)$ & $0.63736(17)$ & $0.82771(13)$ & 0.0214 \\
\hline $\mathrm{H} 4 \mathrm{~N}$ & 0.9133 & 0.5838 & 0.8284 & $0.026^{*}$ \\
\hline N5 & $0.4566(2)$ & $0.88624(18)$ & $0.63630(16)$ & $0.0303(4)$ \\
\hline N6 & $0.3789(2)$ & $0.8477(2)$ & $0.57414(18)$ & $0.0394(5)$ \\
\hline N7 & $0.7377(2)$ & $0.40229(17)$ & $0.58472(14)$ & $0.0247(4)$ \\
\hline N8 & $0.8855(2)$ & $0.28937(17)$ & $0.59920(14)$ & $0.0243(4)$ \\
\hline N9 & $0.9977(2)$ & $0.32420(18)$ & $0.63093(16)$ & $0.0298(4)$ \\
\hline N10 & $0.9232(2)$ & $0.46781(18)$ & $0.63767(15)$ & $0.0280(4)$ \\
\hline $\mathrm{C} 1$ & $1.2673(3)$ & $-0.1457(2)$ & $1.11176(19)$ & $0.0308(5)$ \\
\hline $\mathrm{H} 1 \mathrm{~A}$ & 1.3074 & -0.1629 & 1.0333 & $0.046^{*}$ \\
\hline H1B & 1.2544 & -0.2373 & 1.1643 & $0.046^{*}$ \\
\hline $\mathrm{H} 1 \mathrm{C}$ & 1.3512 & -0.1201 & 1.1355 & $0.046^{*}$ \\
\hline $\mathrm{C} 2$ & $1.0957(2)$ & $0.1075(2)$ & $1.04081(16)$ & $0.0228(4)$ \\
\hline $\mathrm{C} 3$ & $1.2338(2)$ & $0.1330(2)$ & $0.96351(17)$ & $0.0262(4)$ \\
\hline H3 & 1.3452 & 0.0575 & 0.9589 & $0.031 *$ \\
\hline $\mathrm{C} 4$ & $1.1988(2)$ & $0.2757(2)$ & $0.89353(17)$ & $0.0244(4)$ \\
\hline
\end{tabular}
torsion angles; correlations between e.s.d.'s in cell parameters are only used when they are defined by crystal symmetry. An approximate (isotropic) treatment of cell e.s.d.'s is used for estimating e.s.d.'s involving 1.s. planes.

Fractional atomic coordinates and isotropic or equivalent isotropic displacement parameters $\left(\AA^{2}\right)$ 
supporting information

$\begin{array}{lllll}\text { C5 } & 0.9207(2) & 0.3420(2) & 0.97398(16) & 0.0215(4) \\ \text { C6 } & 1.2928(3) & 0.4514(3) & 0.7412(2) & 0.0393(6) \\ \text { H6A } & 1.2067 & 0.4667 & 0.6948 & 0.059^{*} \\ \text { H6B } & 1.3985 & 0.4573 & 0.6904 & 0.059^{*} \\ \text { H6C } & 1.2486 & 0.5297 & 0.7861 & 0.059^{*} \\ \text { C7 } & 0.6855(2) & 0.5842(2) & 0.90723(16) & 0.0231(4) \\ \text { C8 } & 0.6097(2) & 0.7766(2) & 0.65845(16) & 0.0215(4) \\ \text { C9 } & 0.4833(3) & 0.7125(2) & 0.5569(2) & 0.0316(5) \\ \text { H9 } & 0.4601 & 0.6571 & 0.5153 & 0.038^{*} \\ \text { C10 } & 0.6314(2) & 0.6612(2) & 0.60716(16) & 0.0212(4) \\ \text { C11 } & 0.3620(3) & 1.0278(3) & 0.6757(3) & 0.0496(7) \\ \text { H11A } & 0.2815 & 1.0973 & 0.6224 & 0.074^{*} \\ \text { H11B } & 0.4431 & 1.0732 & 0.6783 & 0.074^{*} \\ \text { H11C } & 0.2981 & 1.0074 & 0.7527 & 0.074^{*} \\ \text { C12 } & 0.7652(2) & 0.5127(2) & 0.60905(16) & 0.0204(4) \\ \text { C13 } & 0.9157(3) & 0.1370(2) & 0.5897(2) & 0.0326(5) \\ \text { H13A } & 1.0396 & 0.0807 & 0.5778 & 0.049^{*} \\ \text { H13B } & 0.8653 & 0.1424 & 0.5244 & 0.049^{*} \\ \text { H13C } & 0.8625 & 0.0857 & 0.6606 & 0.049^{*}\end{array}$

Atomic displacement parameters $\left(\AA^{2}\right)$

\begin{tabular}{lllllll}
\hline & $U^{11}$ & $U^{22}$ & $U^{33}$ & $U^{12}$ & $U^{13}$ & $U^{23}$ \\
\hline S1 & $0.0261(3)$ & $0.0177(2)$ & $0.0299(3)$ & $-0.00848(19)$ & $-0.0094(2)$ & $-0.00349(18)$ \\
O1 & $0.0263(7)$ & $0.0221(7)$ & $0.0311(8)$ & $-0.0045(6)$ & $-0.0073(6)$ & $-0.0024(6)$ \\
O2 & $0.0173(7)$ & $0.0365(8)$ & $0.0414(9)$ & $-0.0065(6)$ & $-0.0006(6)$ & $0.0009(7)$ \\
O3 & $0.0177(7)$ & $0.0287(7)$ & $0.0380(8)$ & $-0.0007(6)$ & $0.0005(6)$ & $-0.0025(6)$ \\
O4 & $0.0478(10)$ & $0.0220(7)$ & $0.0494(9)$ & $-0.0038(7)$ & $-0.0234(8)$ & $-0.0146(7)$ \\
O5 & $0.0301(8)$ & $0.0364(8)$ & $0.0425(9)$ & $-0.0205(7)$ & $-0.0075(7)$ & $0.0047(7)$ \\
N1 & $0.0213(8)$ & $0.0249(8)$ & $0.0240(8)$ & $-0.0068(7)$ & $-0.0050(7)$ & $-0.0050(6)$ \\
N2 & $0.0176(8)$ & $0.0255(8)$ & $0.0253(8)$ & $-0.0046(7)$ & $-0.0039(7)$ & $-0.0049(7)$ \\
N3 & $0.0174(8)$ & $0.0254(8)$ & $0.0260(8)$ & $-0.0041(7)$ & $0.0002(7)$ & $-0.0024(7)$ \\
N4 & $0.0162(7)$ & $0.0200(7)$ & $0.0251(8)$ & $-0.0028(6)$ & $-0.0050(6)$ & $-0.0042(6)$ \\
N5 & $0.0265(9)$ & $0.0225(8)$ & $0.0423(10)$ & $0.0018(7)$ & $-0.0164(8)$ & $-0.0132(7)$ \\
N6 & $0.0336(10)$ & $0.0331(10)$ & $0.0538(12)$ & $0.0015(8)$ & $-0.0243(9)$ & $-0.0174(9)$ \\
N7 & $0.0234(8)$ & $0.0194(7)$ & $0.0312(9)$ & $-0.0041(7)$ & $-0.0068(7)$ & $-0.0078(7)$ \\
N8 & $0.0260(8)$ & $0.0182(7)$ & $0.0293(9)$ & $-0.0047(7)$ & $-0.0063(7)$ & $-0.0087(6)$ \\
N9 & $0.0252(9)$ & $0.0236(8)$ & $0.0411(10)$ & $-0.0021(7)$ & $-0.0102(8)$ & $-0.0131(7)$ \\
N10 & $0.0233(8)$ & $0.0212(8)$ & $0.0409(10)$ & $-0.0024(7)$ & $-0.0103(8)$ & $-0.0121(7)$ \\
C1 & $0.0283(11)$ & $0.0193(9)$ & $0.0403(12)$ & $-0.0001(8)$ & $-0.0127(9)$ & $-0.0055(8)$ \\
C2 & $0.0234(10)$ & $0.0227(9)$ & $0.0236(9)$ & $-0.0058(8)$ & $-0.0094(8)$ & $-0.0056(7)$ \\
C3 & $0.0180(9)$ & $0.0271(10)$ & $0.0298(10)$ & $-0.0027(8)$ & $-0.0066(8)$ & $-0.0060(8)$ \\
C4 & $0.0165(9)$ & $0.0297(10)$ & $0.0262(10)$ & $-0.0071(8)$ & $-0.0042(8)$ & $-0.0056(8)$ \\
C5 & $0.0173(9)$ & $0.0246(9)$ & $0.0228(9)$ & $-0.0049(8)$ & $-0.0044(7)$ & $-0.0076(7)$ \\
C6 & $0.0283(11)$ & $0.0411(13)$ & $0.0386(13)$ & $-0.0145(10)$ & $0.0007(10)$ & $0.0043(10)$ \\
C7 & $0.0200(9)$ & $0.0242(9)$ & $0.0230(9)$ & $-0.0051(8)$ & $-0.0010(8)$ & $-0.0075(7)$ \\
C8 & $0.0213(9)$ & $0.0172(8)$ & $0.0249(9)$ & $-0.0028(7)$ & $-0.0079(8)$ & $-0.0050(7)$ \\
C9 & $0.0297(11)$ & $0.0264(10)$ & $0.0419(12)$ & $-0.0028(9)$ & $-0.0170(10)$ & $-0.0124(9)$ \\
& & & & & &
\end{tabular}




\begin{tabular}{lllllll}
$\mathrm{C} 10$ & $0.0209(9)$ & $0.0187(9)$ & $0.0237(9)$ & $-0.0057(7)$ & $-0.0056(8)$ & $-0.0040(7)$ \\
$\mathrm{C} 11$ & $0.0405(14)$ & $0.0317(12)$ & $0.0737(18)$ & $0.0128(11)$ & $-0.0255(13)$ & $-0.0302(12)$ \\
$\mathrm{C} 12$ & $0.0206(9)$ & $0.0198(8)$ & $0.0219(9)$ & $-0.0056(7)$ & $-0.0053(7)$ & $-0.0066(7)$ \\
$\mathrm{C} 13$ & $0.0393(12)$ & $0.0181(9)$ & $0.0415(12)$ & $-0.0063(9)$ & $-0.0074(10)$ & $-0.0125(8)$ \\
\hline
\end{tabular}

Geometric parameters $\left(A,{ }^{\circ}\right)$

\begin{tabular}{|c|c|c|c|}
\hline $\mathrm{S} 1-\mathrm{O} 4$ & $1.4201(15)$ & $\mathrm{N} 8-\mathrm{N} 9$ & $1.314(2)$ \\
\hline $\mathrm{S} 1-\mathrm{O} 5$ & $1.4243(16)$ & $\mathrm{N} 8-\mathrm{C} 13$ & $1.462(2)$ \\
\hline $\mathrm{S} 1-\mathrm{N} 4$ & $1.6284(16)$ & N9-N10 & $1.325(2)$ \\
\hline $\mathrm{S} 1-\mathrm{C} 8$ & $1.751(2)$ & $\mathrm{N} 10-\mathrm{C} 12$ & $1.350(2)$ \\
\hline $\mathrm{O} 1-\mathrm{C} 2$ & $1.338(2)$ & $\mathrm{C} 1-\mathrm{H} 1 \mathrm{~A}$ & 0.9800 \\
\hline $\mathrm{O} 1-\mathrm{C} 1$ & $1.444(2)$ & $\mathrm{C} 1-\mathrm{H} 1 \mathrm{~B}$ & 0.9800 \\
\hline $\mathrm{O} 2-\mathrm{C} 4$ & $1.337(2)$ & $\mathrm{C} 1-\mathrm{H} 1 \mathrm{C}$ & 0.9800 \\
\hline $\mathrm{O} 2-\mathrm{C} 6$ & $1.444(3)$ & $\mathrm{C} 2-\mathrm{C} 3$ & $1.387(3)$ \\
\hline $\mathrm{O} 3-\mathrm{C} 7$ & $1.217(2)$ & $\mathrm{C} 3-\mathrm{C} 4$ & $1.383(3)$ \\
\hline $\mathrm{N} 1-\mathrm{C} 5$ & $1.321(2)$ & $\mathrm{C} 3-\mathrm{H} 3$ & 0.9500 \\
\hline $\mathrm{N} 1-\mathrm{C} 2$ & $1.341(2)$ & C6-H6A & 0.9800 \\
\hline $\mathrm{N} 2-\mathrm{C} 4$ & $1.330(2)$ & C6-H6B & 0.9800 \\
\hline $\mathrm{N} 2-\mathrm{C} 5$ & $1.342(2)$ & $\mathrm{C} 6-\mathrm{H} 6 \mathrm{C}$ & 0.9800 \\
\hline $\mathrm{N} 3-\mathrm{C} 7$ & $1.373(2)$ & $\mathrm{C} 8-\mathrm{C} 10$ & $1.389(3)$ \\
\hline $\mathrm{N} 3-\mathrm{C} 5$ & $1.397(2)$ & $\mathrm{C} 9-\mathrm{C} 10$ & $1.393(3)$ \\
\hline $\mathrm{N} 3-\mathrm{H} 3 \mathrm{~N}$ & 0.8800 & $\mathrm{C} 9-\mathrm{H} 9$ & 0.9500 \\
\hline $\mathrm{N} 4-\mathrm{C} 7$ & $1.378(2)$ & $\mathrm{C} 10-\mathrm{C} 12$ & $1.459(2)$ \\
\hline $\mathrm{N} 4-\mathrm{H} 4 \mathrm{~N}$ & 0.8800 & $\mathrm{C} 11-\mathrm{H} 11 \mathrm{~A}$ & 0.9800 \\
\hline N5-N6 & $1.340(3)$ & C11-H11B & 0.9800 \\
\hline N5-C8 & $1.358(2)$ & $\mathrm{C} 11-\mathrm{H} 11 \mathrm{C}$ & 0.9800 \\
\hline N5-C11 & $1.466(3)$ & $\mathrm{C} 13-\mathrm{H} 13 \mathrm{~A}$ & 0.9800 \\
\hline $\mathrm{N} 6-\mathrm{C} 9$ & $1.325(3)$ & C13-H13B & 0.9800 \\
\hline $\mathrm{N} 7-\mathrm{N} 8$ & $1.324(2)$ & $\mathrm{C} 13-\mathrm{H} 13 \mathrm{C}$ & 0.9800 \\
\hline $\mathrm{N} 7-\mathrm{C} 12$ & $1.332(2)$ & & \\
\hline $\mathrm{O} 4-\mathrm{S} 1-\mathrm{O} 5$ & $119.58(10)$ & $\mathrm{N} 2-\mathrm{C} 4-\mathrm{C} 3$ & $123.37(18)$ \\
\hline $\mathrm{O} 4-\mathrm{S} 1-\mathrm{N} 4$ & $109.86(9)$ & $\mathrm{O} 2-\mathrm{C} 4-\mathrm{C} 3$ & $117.43(17)$ \\
\hline $\mathrm{O} 5-\mathrm{S} 1-\mathrm{N} 4$ & $104.30(9)$ & $\mathrm{N} 1-\mathrm{C} 5-\mathrm{N} 2$ & $127.89(17)$ \\
\hline $\mathrm{O} 4-\mathrm{S} 1-\mathrm{C} 8$ & $107.79(9)$ & $\mathrm{N} 1-\mathrm{C} 5-\mathrm{N} 3$ & $114.04(17)$ \\
\hline $\mathrm{O} 5-\mathrm{S} 1-\mathrm{C} 8$ & $109.46(10)$ & $\mathrm{N} 2-\mathrm{C} 5-\mathrm{N} 3$ & $118.05(16)$ \\
\hline $\mathrm{N} 4-\mathrm{S} 1-\mathrm{C} 8$ & $104.90(9)$ & $\mathrm{O} 2-\mathrm{C} 6-\mathrm{H} 6 \mathrm{~A}$ & 109.5 \\
\hline $\mathrm{C} 2-\mathrm{O} 1-\mathrm{C} 1$ & $117.09(16)$ & $\mathrm{O} 2-\mathrm{C} 6-\mathrm{H} 6 \mathrm{~B}$ & 109.5 \\
\hline $\mathrm{C} 4-\mathrm{O} 2-\mathrm{C} 6$ & $118.04(16)$ & $\mathrm{H} 6 \mathrm{~A}-\mathrm{C} 6-\mathrm{H} 6 \mathrm{~B}$ & 109.5 \\
\hline $\mathrm{C} 5-\mathrm{N} 1-\mathrm{C} 2$ & $114.99(17)$ & $\mathrm{O} 2-\mathrm{C} 6-\mathrm{H} 6 \mathrm{C}$ & 109.5 \\
\hline $\mathrm{C} 4-\mathrm{N} 2-\mathrm{C} 5$ & $114.96(16)$ & $\mathrm{H} 6 \mathrm{~A}-\mathrm{C} 6-\mathrm{H} 6 \mathrm{C}$ & 109.5 \\
\hline $\mathrm{C} 7-\mathrm{N} 3-\mathrm{C} 5$ & $129.98(17)$ & $\mathrm{H} 6 \mathrm{~B}-\mathrm{C} 6-\mathrm{H} 6 \mathrm{C}$ & 109.5 \\
\hline $\mathrm{C} 7-\mathrm{N} 3-\mathrm{H} 3 \mathrm{~N}$ & 115.0 & $\mathrm{O} 3-\mathrm{C} 7-\mathrm{N} 3$ & $121.66(18)$ \\
\hline $\mathrm{C} 5-\mathrm{N} 3-\mathrm{H} 3 \mathrm{~N}$ & 115.0 & $\mathrm{O} 3-\mathrm{C} 7-\mathrm{N} 4$ & $122.49(17)$ \\
\hline $\mathrm{C} 7-\mathrm{N} 4-\mathrm{S} 1$ & $123.00(13)$ & $\mathrm{N} 3-\mathrm{C} 7-\mathrm{N} 4$ & $115.85(16)$ \\
\hline $\mathrm{C} 7-\mathrm{N} 4-\mathrm{H} 4 \mathrm{~N}$ & 118.5 & $\mathrm{~N} 5-\mathrm{C} 8-\mathrm{C} 10$ & $107.00(17)$ \\
\hline $\mathrm{S} 1-\mathrm{N} 4-\mathrm{H} 4 \mathrm{~N}$ & 118.5 & $\mathrm{~N} 5-\mathrm{C} 8-\mathrm{S} 1$ & $122.90(14)$ \\
\hline
\end{tabular}




\begin{tabular}{|c|c|c|c|}
\hline $\mathrm{N} 6-\mathrm{N} 5-\mathrm{C} 8$ & $111.29(16)$ & $\mathrm{C} 10-\mathrm{C} 8-\mathrm{S} 1$ & $130.02(14)$ \\
\hline $\mathrm{N} 6-\mathrm{N} 5-\mathrm{C} 11$ & $117.40(17)$ & $\mathrm{N} 6-\mathrm{C} 9-\mathrm{C} 10$ & $111.85(19)$ \\
\hline $\mathrm{C} 8-\mathrm{N} 5-\mathrm{C} 11$ & $131.12(18)$ & $\mathrm{N} 6-\mathrm{C} 9-\mathrm{H} 9$ & 124.1 \\
\hline $\mathrm{C} 9-\mathrm{N} 6-\mathrm{N} 5$ & $105.78(17)$ & $\mathrm{C} 10-\mathrm{C} 9-\mathrm{H} 9$ & 124.1 \\
\hline $\mathrm{N} 8-\mathrm{N} 7-\mathrm{C} 12$ & $101.76(15)$ & $\mathrm{C} 8-\mathrm{C} 10-\mathrm{C} 9$ & $104.07(16)$ \\
\hline N9-N8-N7 & $113.84(15)$ & $\mathrm{C} 8-\mathrm{C} 10-\mathrm{C} 12$ & $131.15(18)$ \\
\hline $\mathrm{N} 9-\mathrm{N} 8-\mathrm{C} 13$ & $123.11(16)$ & $\mathrm{C} 9-\mathrm{C} 10-\mathrm{C} 12$ & $124.59(18)$ \\
\hline $\mathrm{N} 7-\mathrm{N} 8-\mathrm{C} 13$ & $122.87(17)$ & N5-C11-H11A & 109.5 \\
\hline $\mathrm{N} 8-\mathrm{N} 9-\mathrm{N} 10$ & $106.55(15)$ & N5-C11-H11B & 109.5 \\
\hline $\mathrm{N} 9-\mathrm{N} 10-\mathrm{C} 12$ & $105.48(16)$ & $\mathrm{H} 11 \mathrm{~A}-\mathrm{C} 11-\mathrm{H} 11 \mathrm{~B}$ & 109.5 \\
\hline $\mathrm{O} 1-\mathrm{C} 1-\mathrm{H} 1 \mathrm{~A}$ & 109.5 & $\mathrm{~N} 5-\mathrm{C} 11-\mathrm{H} 11 \mathrm{C}$ & 109.5 \\
\hline $\mathrm{O} 1-\mathrm{C} 1-\mathrm{H} 1 \mathrm{~B}$ & 109.5 & $\mathrm{H} 11 \mathrm{~A}-\mathrm{C} 11-\mathrm{H} 11 \mathrm{C}$ & 109.5 \\
\hline $\mathrm{H} 1 \mathrm{~A}-\mathrm{C} 1-\mathrm{H} 1 \mathrm{~B}$ & 109.5 & $\mathrm{H} 11 \mathrm{~B}-\mathrm{C} 11-\mathrm{H} 11 \mathrm{C}$ & 109.5 \\
\hline $\mathrm{O} 1-\mathrm{C} 1-\mathrm{H} 1 \mathrm{C}$ & 109.5 & $\mathrm{~N} 7-\mathrm{C} 12-\mathrm{N} 10$ & $112.36(16)$ \\
\hline $\mathrm{H} 1 \mathrm{~A}-\mathrm{C} 1-\mathrm{H} 1 \mathrm{C}$ & 109.5 & $\mathrm{~N} 7-\mathrm{C} 12-\mathrm{C} 10$ & $121.13(17)$ \\
\hline $\mathrm{H} 1 \mathrm{~B}-\mathrm{C} 1-\mathrm{H} 1 \mathrm{C}$ & 109.5 & $\mathrm{~N} 10-\mathrm{C} 12-\mathrm{C} 10$ & $126.48(17)$ \\
\hline $\mathrm{O} 1-\mathrm{C} 2-\mathrm{N} 1$ & $112.43(17)$ & $\mathrm{N} 8-\mathrm{C} 13-\mathrm{H} 13 \mathrm{~A}$ & 109.5 \\
\hline $\mathrm{O} 1-\mathrm{C} 2-\mathrm{C} 3$ & $124.43(17)$ & $\mathrm{N} 8-\mathrm{C} 13-\mathrm{H} 13 \mathrm{~B}$ & 109.5 \\
\hline $\mathrm{N} 1-\mathrm{C} 2-\mathrm{C} 3$ & $123.14(18)$ & $\mathrm{H} 13 \mathrm{~A}-\mathrm{C} 13-\mathrm{H} 13 \mathrm{~B}$ & 109.5 \\
\hline $\mathrm{C} 4-\mathrm{C} 3-\mathrm{C} 2$ & $115.60(17)$ & $\mathrm{N} 8-\mathrm{C} 13-\mathrm{H} 13 \mathrm{C}$ & 109.5 \\
\hline $\mathrm{C} 4-\mathrm{C} 3-\mathrm{H} 3$ & 122.2 & $\mathrm{H} 13 \mathrm{~A}-\mathrm{C} 13-\mathrm{H} 13 \mathrm{C}$ & 109.5 \\
\hline $\mathrm{C} 2-\mathrm{C} 3-\mathrm{H} 3$ & 122.2 & $\mathrm{H} 13 \mathrm{~B}-\mathrm{C} 13-\mathrm{H} 13 \mathrm{C}$ & 109.5 \\
\hline $\mathrm{N} 2-\mathrm{C} 4-\mathrm{O} 2$ & $119.19(17)$ & & \\
\hline $\mathrm{O} 4-\mathrm{S} 1-\mathrm{N} 4-\mathrm{C} 7$ & $59.80(18)$ & $\mathrm{C} 5-\mathrm{N} 3-\mathrm{C} 7-\mathrm{N} 4$ & $-7.5(3)$ \\
\hline $\mathrm{O} 5-\mathrm{S} 1-\mathrm{N} 4-\mathrm{C} 7$ & $-170.87(15)$ & $\mathrm{S} 1-\mathrm{N} 4-\mathrm{C} 7-\mathrm{O} 3$ & $-2.7(3)$ \\
\hline $\mathrm{C} 8-\mathrm{S} 1-\mathrm{N} 4-\mathrm{C} 7$ & $-55.81(17)$ & $\mathrm{S} 1-\mathrm{N} 4-\mathrm{C} 7-\mathrm{N} 3$ & $176.66(13)$ \\
\hline $\mathrm{C} 8-\mathrm{N} 5-\mathrm{N} 6-\mathrm{C} 9$ & $0.1(3)$ & $\mathrm{N} 6-\mathrm{N} 5-\mathrm{C} 8-\mathrm{C} 10$ & $0.1(2)$ \\
\hline $\mathrm{C} 11-\mathrm{N} 5-\mathrm{N} 6-\mathrm{C} 9$ & $-175.4(2)$ & $\mathrm{C} 11-\mathrm{N} 5-\mathrm{C} 8-\mathrm{C} 10$ & $174.9(2)$ \\
\hline $\mathrm{C} 12-\mathrm{N} 7-\mathrm{N} 8-\mathrm{N} 9$ & $0.4(2)$ & $\mathrm{N} 6-\mathrm{N} 5-\mathrm{C} 8-\mathrm{S} 1$ & $177.18(16)$ \\
\hline $\mathrm{C} 12-\mathrm{N} 7-\mathrm{N} 8-\mathrm{C} 13$ & $175.78(18)$ & $\mathrm{C} 11-\mathrm{N} 5-\mathrm{C} 8-\mathrm{S} 1$ & $-8.1(3)$ \\
\hline $\mathrm{N} 7-\mathrm{N} 8-\mathrm{N} 9-\mathrm{N} 10$ & $-0.4(2)$ & $\mathrm{O} 4-\mathrm{S} 1-\mathrm{C} 8-\mathrm{N} 5$ & $15.8(2)$ \\
\hline $\mathrm{C} 13-\mathrm{N} 8-\mathrm{N} 9-\mathrm{N} 10$ & $-175.73(18)$ & $\mathrm{O} 5-\mathrm{S} 1-\mathrm{C} 8-\mathrm{N} 5$ & $-115.78(18)$ \\
\hline $\mathrm{N} 8-\mathrm{N} 9-\mathrm{N} 10-\mathrm{C} 12$ & $0.2(2)$ & $\mathrm{N} 4-\mathrm{S} 1-\mathrm{C} 8-\mathrm{N} 5$ & $132.80(17)$ \\
\hline $\mathrm{C} 1-\mathrm{O} 1-\mathrm{C} 2-\mathrm{N} 1$ & $177.72(17)$ & $\mathrm{O} 4-\mathrm{S} 1-\mathrm{C} 8-\mathrm{C} 10$ & $-167.94(18)$ \\
\hline $\mathrm{C} 1-\mathrm{O} 1-\mathrm{C} 2-\mathrm{C} 3$ & $-1.3(3)$ & $\mathrm{O} 5-\mathrm{S} 1-\mathrm{C} 8-\mathrm{C} 10$ & $60.5(2)$ \\
\hline $\mathrm{C} 5-\mathrm{N} 1-\mathrm{C} 2-\mathrm{O} 1$ & $-178.07(16)$ & $\mathrm{N} 4-\mathrm{S} 1-\mathrm{C} 8-\mathrm{C} 10$ & $-50.9(2)$ \\
\hline $\mathrm{C} 5-\mathrm{N} 1-\mathrm{C} 2-\mathrm{C} 3$ & $1.0(3)$ & $\mathrm{N} 5-\mathrm{N} 6-\mathrm{C} 9-\mathrm{C} 10$ & $-0.4(3)$ \\
\hline $\mathrm{O} 1-\mathrm{C} 2-\mathrm{C} 3-\mathrm{C} 4$ & $179.64(18)$ & $\mathrm{N} 5-\mathrm{C} 8-\mathrm{C} 10-\mathrm{C} 9$ & $-0.3(2)$ \\
\hline $\mathrm{N} 1-\mathrm{C} 2-\mathrm{C} 3-\mathrm{C} 4$ & $0.7(3)$ & $\mathrm{S} 1-\mathrm{C} 8-\mathrm{C} 10-\mathrm{C} 9$ & $-177.09(17)$ \\
\hline $\mathrm{C} 5-\mathrm{N} 2-\mathrm{C} 4-\mathrm{O} 2$ & $178.90(17)$ & $\mathrm{N} 5-\mathrm{C} 8-\mathrm{C} 10-\mathrm{C} 12$ & $-175.5(2)$ \\
\hline $\mathrm{C} 5-\mathrm{N} 2-\mathrm{C} 4-\mathrm{C} 3$ & $-0.2(3)$ & $\mathrm{S} 1-\mathrm{C} 8-\mathrm{C} 10-\mathrm{C} 12$ & $7.8(3)$ \\
\hline $\mathrm{C} 6-\mathrm{O} 2-\mathrm{C} 4-\mathrm{N} 2$ & $-1.7(3)$ & $\mathrm{N} 6-\mathrm{C} 9-\mathrm{C} 10-\mathrm{C} 8$ & $0.4(3)$ \\
\hline $\mathrm{C} 6-\mathrm{O} 2-\mathrm{C} 4-\mathrm{C} 3$ & $177.50(19)$ & $\mathrm{N} 6-\mathrm{C} 9-\mathrm{C} 10-\mathrm{C} 12$ & $176.0(2)$ \\
\hline $\mathrm{C} 2-\mathrm{C} 3-\mathrm{C} 4-\mathrm{N} 2$ & $-1.1(3)$ & $\mathrm{N} 8-\mathrm{N} 7-\mathrm{C} 12-\mathrm{N} 10$ & $-0.3(2)$ \\
\hline $\mathrm{C} 2-\mathrm{C} 3-\mathrm{C} 4-\mathrm{O} 2$ & $179.76(18)$ & $\mathrm{N} 8-\mathrm{N} 7-\mathrm{C} 12-\mathrm{C} 10$ & $-178.32(17)$ \\
\hline $\mathrm{C} 2-\mathrm{N} 1-\mathrm{C} 5-\mathrm{N} 2$ & $-2.7(3)$ & $\mathrm{N} 9-\mathrm{N} 10-\mathrm{C} 12-\mathrm{N} 7$ & $0.1(2)$ \\
\hline $\mathrm{C} 2-\mathrm{N} 1-\mathrm{C} 5-\mathrm{N} 3$ & $175.99(17)$ & $\mathrm{N} 9-\mathrm{N} 10-\mathrm{C} 12-\mathrm{C} 10$ & $177.97(18)$ \\
\hline
\end{tabular}




$\begin{array}{lllr}\mathrm{C} 4-\mathrm{N} 2-\mathrm{C} 5-\mathrm{N} 1 & 2.3(3) & \mathrm{C} 8-\mathrm{C} 10-\mathrm{C} 12-\mathrm{N} 7 & 159.2(2) \\ \mathrm{C} 4-\mathrm{N} 2-\mathrm{C} 5-\mathrm{N} 3 & -176.29(17) & \mathrm{C} 9-\mathrm{C} 10-\mathrm{C} 12-\mathrm{N} 7 & -15.0(3) \\ \mathrm{C} 7-\mathrm{N} 3-\mathrm{C} 5-\mathrm{N} 1 & -173.08(19) & \mathrm{C} 8-\mathrm{C} 10-\mathrm{C} 12-\mathrm{N} 10 & -18.5(3) \\ \mathrm{C} 7-\mathrm{N} 3-\mathrm{C} 5-\mathrm{N} 2 & 5.7(3) & \mathrm{C} 9-\mathrm{C} 10-\mathrm{C} 12-\mathrm{N} 10 & 167.3(2) \\ \mathrm{C} 5-\mathrm{N} 3-\mathrm{C} 7-\mathrm{O} 3 & 171.9(2) & & \end{array}$

Hydrogen-bond geometry $\left(\AA,{ }^{\circ}\right)$

\begin{tabular}{lllll}
\hline$D-\mathrm{H} \cdots A$ & $D-\mathrm{H}$ & $\mathrm{H} \cdots A$ & $D \cdots A$ & $D-\mathrm{H} \cdots A$ \\
\hline $\mathrm{N} 3-\mathrm{H} 3 N \cdots \mathrm{O} 3^{\mathrm{i}}$ & 0.88 & 2.09 & $2.877(2)$ & 149 \\
$\mathrm{C} 1-\mathrm{H} 1 A \cdots \mathrm{O} 3^{\text {ii }}$ & 0.98 & 2.55 & $3.381(3)$ & 143 \\
$\mathrm{C} 1-\mathrm{H} 1 C \cdots \mathrm{O} 4^{\text {iii }}$ & 0.98 & 2.44 & $3.225(3)$ & 137 \\
$\mathrm{C} 11-\mathrm{H} 11 A \cdots \mathrm{N}^{\text {iv }}$ & 0.98 & 2.57 & $3.357(3)$ & 137 \\
$\mathrm{C} 13-\mathrm{H} 13 B \cdots{ }^{2}$ & 0.98 & 2.62 & $3.533(3)$ & 155 \\
\hline
\end{tabular}

Symmetry codes: (i) $-x+1,-y+1,-z+2$; (ii) $x+1, y-1, z$; (iii) $-x+2,-y+1,-z+2$; (iv) $x-1, y+1, z$; (v) $-x+1,-y+1,-z+1$. 